\title{
INNOVACIÓN DIDÁCTICA INCLUSIVA BASADA EN LA PEDAGOGÍA DIALÓGICA PARTICIPATIVA E INTERACTIVA APLICADA AL ÁREA DE MATEMÁTICA EN ESTUDIANTE VULNERABLE BASADA EN UN ESTUDIO DE CASO
}

INNOVACIÓN DIDÁCTICA INCLUSIVA BASADA EN LA PEDAGOGÍA DIALÓGICA PARTICIPATIVA E INTERACTIVA APLICADA AL ÁREA DE MATEMÁTICA EN ESTUDIANTE VULNERABLE BASADA EN UN ESTUDIO DE CASO

IGNACIO EDUARDO CAYO PIZARRO ${ }^{1}$ BETTY SHADIRA PROCEL GUERRA²

Recibido: 18 de junio de 2018 Aceptado: 22 de octubre de 2018

\footnotetext{
${ }^{1}$ Universidad Diego Portales, Chile, icayop@gmail.com

${ }^{2}$ Chile, bshpg@hotmail.com
} 
ill|li4

214 


\title{
INNOVACIÓN DIDÁCTICA INCLUSIVA BASADA EN LA PEDAGOGÍA DIALÓGICA PARTICIPATIVA E INTERACTIVA APLICADA AL ÁREA DE MATEMÁTICA EN ESTUDIANTE VULNERABLE BASADA EN UN ESTUDIO DE CASO
}

INNOVACIÓN DIDÁCTICA INCLUSIVA BASADA EN LA PEDAGOGÍA DIALÓGICA PARTICIPATIVA E INTERACTIVA APLICADA AL ÁREA DE MATEMÁTICA EN ESTUDIANTE VULNERABLE BASADA EN UN ESTUDIO DE CASO

\author{
Ignacio Eduardo Cayo Pizarro, Betty Shadira Procel Guerra
}

Palabras clave: Innovación didáctica, Pedagogía dialógica, estudiantes vulnerables, didáctica inclusiva

Key words: Didactic innovation, dialogic pedagogy, vulnerable students, inclusive didactics 


\section{RESUMEN}

La propuesta de innovación didáctica se contextualiza en los modelos dialógicos, participativos e interactivos que favorecen el aprendizaje de los estudiantes. La metodología de trabajo se posicionó en la investigación acción participativa y se estructura en colaboración entre educadores y estudiantes de un colegio subvencionado con altos niveles de vulnerabilidad en la Comuna Coronel-Chile. El diseño de innovación estableció estaciones de aprendizaje utilizando la motricidad, concentración y desarrollo de habilidades de razonamiento lógico matemático. El modelo de innovación didáctica se denominó "Yincana de las Matemáticas", en el cual los estudiantes fueron actores protagónicos, retroalimentando el modelo para su posterior reformulación mediante el diálogo con los educandos. Los resultados evidenciaron que los estudiantes tuvieron, ante este tipo de iniciativas, una activa interacción entre sus compañeros y fomentaron la integración e inclusión. Además, la iniciativa potenció el trabajo colaborativo entre los educadores (educadora diferencial, profesores jefes, profesor de matemática y orientador educacional), mejorando el ambiente de aprendizaje y clima de aula. Por lo tanto, los aprendizajes desarrollados permitieron que los estudiantes fueran los principales evaluadores de la actividad.

\section{ABSTRACT}

The didactic innovation proposal is contextualized in the dialogical, participatory and interactive models that favor student learning. The work methodology was positioned in participatory action research and is structured in collaboration between educators and students of a subsidized school with high levels of vulnerability in the Comuna Coronel-Chile. The innovation design established learning stations using the motor, concentration and development of mathematical logical reasoning skills. The didactic innovation model was called "Yincana de las Mathematicas", in which the students were protagonists, feeding back the model for its subsequent reformulation through dialogue with the students. The results showed that the students had, before this type of initiative, an active interaction among their peers and encouraged integration and inclusion. In addition, the initiative strengthened the collaborative work 
among educators (differential educator, chief teachers, mathematics teacher and educational counselor), improving the learning environment and classroom cli- mate. Therefore, the developed learning allowed students to be the main evaluators of the activity.

\section{INTRODUCCIÓN}

El establecimiento educacional intervenido se encuentra localizado en la Comuna de Coronel, Octava región. El entorno del sitio presenta aspectos de depresión cultural, económica y social, tiene un índice de vulneración del 89\% y más del $60 \%$ de los padres y madres no han terminado su enseñanza formal. El proyecto de innovación buscaba generar espacios de diálogo y democratizar los procesos de enseñanza. Sin embargo, los aspectos familiares y los modelos educacionales no proponen diálogos transversales, ni consulta a los estudiantes cómo les gustaría que fuera su clase y la forma de aprender (Abellán, 2011). Este proyecto de innovación fue una herramienta efectiva para incentivar el diálogo transversal entre estudiantes, profesores, equipo del Proyecto de Integración Educativo (P.I.E) y equipo directivo.

Los estudiantes de los $6^{\circ}$ básicos de dicho establecimiento educativo manifestaron, en reiteradas ocasiones, la necesidad de mayor dinamismo en las clases de matemáticas. El descontento mostrado por los estudiantes por cómo se llevaban las clases generó múltiples dificultades por parte de los docentes al momento de realizarlas. Durante la clase de orientación, se condujo un plan de intervención en los cursos ya mencionados. El área de orientación gestionó junto con los profesores un diseño de innovación pedagógica de carácter multidisciplinario. El diseño de innovación incorporó a varios actores educativos, entre los que estaban la educadora diferencial, el/ la profesora(a) de matemática, el orientador educacional y los estudiantes.

Los cursos intervenidos evidenciaron dificultades entre la relación profesor-estudiante, lo que derivó en la ausencia total de los procesos de aprendizaje. Este factor fue evidente cuando en dicho establecimiento se realizaron mediciones de autoevaluación externa de aprendizaje, las mismas que son utilizadas para proyectar futuros resultados de pruebas estandarizadas (SIMCE) y proponer estrategias de mejora en el aula. A raíz de los bajos resultados durante el primer semestre del 2015, se complementó el diseño de innovación con 
material y/o guías de formato estandarizado, con el propósito de generar una mejora continua.

Por lo antes mencionado, el objetivo de este trabajo fue implementar una propuesta didáctica, dialógica/participativa, democratización de las enseñanzas para fomentar las actividades de aprendizaje y potenciar las habilidades de razonamiento lógico matemático en estudiantes $6^{\circ}$ básico. En detalle, esta innovación pedagógica plantea lo siguiente: a) Planificar propuestas didácticas interactivas en $6^{\circ}$ básico, con la colaboración de un equipo multidisciplinario integrado por Proyecto Integración Educativa-profesor(a) dentro de la asignatura de Orientación; b) Diseñar propuestas didácticas en la asignatura de matemática, con una mirada dialógica/participativa entre PIE-profesor(a) de asignatura, estudiantes y orientación; c) Ejecutar la propuesta didáctica dialógica/participativa en los $6^{\circ}$ de básica; d) Evaluar la propuesta didáctica dialógica/participativa, mediante focos grupales con los estudiantes, profesores y educadora diferencial, con la finalidad de rescatar la percepción del estudiantado

\section{CONTEXTO TEÓRICO}

Dentro del ámbito de vulneración cultural, económica y social que se ven inmersos los colegios municipalizados o subvencionados en Chile, se alberga elementos simbólicos y patrimoniales de privación cultural. Bajo esta visión, se genera la reproducción de elementos privativos culturales y sociales que se expresan en el aula. Dicho simbolismo estaría asociado con elementos de sociabilización pedagógica tanto de los adultos como de los estudiantes. Un proceso de internalización genera elementos cognitivos y emocionales que provocan un conjunto de sistemas de hábitos estructurales que dan respuesta a los intereses tanto de los estudiantes como de los educadores. En definitiva, mediante los procesos de contextualización se producen fenómenos de anclaje de meta- cognición de los aprendizajes adquiridos. Este proceso provoca un cultural local de la adquisición de los aprendizajes, expresado así:

entendemos por cultura las capacidades, los valores, los contenidos y los métodos/procedimientos que utiliza una sociedad determinada, que son transmitido de una generación a otra a través de la educación formal o no formal. Estos cuatros 
elementos (capacidad, valores, contenidos y métodos/procedimientos) cuando son interiorizados por el aprendiz. (Román, Díaz, 2009, p. 134).

Los procesos de apertura transversales dentro del aula generan una ruptura de los métodos de condicionamiento sutil e invisible de colegios que están insertos en sectores de vulneración económica y social (Van Dijk, 1994; Torres, 1996). Por lo tanto, en las interacciones pedagógicas dentro de los contextos culturales y sociales de los colegios se requiere una perspectiva distinta del docente y de carácter socio-pedagógico. Como afirma Medrano (1995) "no podemos permitirnos un educador por educando, y aún en este supuesto, no podemos hablar de verdadero aprendizaje sin considerar las relaciones entre compañeros en las interacciones educativas" (p. 178). Los espacios pedagógicos contextualizados en lugares vulnerables requieren dos formas de mediación una social y la otra cultural. Las intervenciones pedagógicas son de carácter organizada y vinculadas a herramientas y signos de aprendizaje de carácter cultural formativo y pedagógico de interacción significativa en el aula (Román y Díaz, 1999; Rodríguez, 1997).

Los establecimientos educacionales se han ido re-construyendo en espacios sociales cada vez más heterogé- neos y con niveles de complejidad cada vez mayores. Por tal motivo, el re-pensar la enseñanza-aprendizaje de las clases, exige un re-conocimiento de las nociones culturales, simbólicas y democráticas que se han instalado en la sociedad. Este contexto enmarcado en una cultura escolar y juvenil demanda mayor participación en las decisiones de cómo aprender las asignaturas (ej.: matemática) (Molina y Sandoval, 2006; Fernández, 2010).

Según Salazar (2011), la labor del educador, bajo los nuevos contextos sociales que experimentan los estudiantes, requiere que sea formado para pensar materia sin duda (enseñar las ciencias occidentales), pero sobre todo para investigar la realidad local que rodea el proceso educativo y para gestionar, dentro y fuera del aula, el desarrollo efectivo de esa realidad. El profesor no puede dejar de ser un investigador, un autor que pública y debate públicamente esa misma investigación (puede enseñar mejor haciendo es) y un profesional que se incorpora a la comunidad real como gestor socio-cultural de su desarrollo efectivo (p. 96).

\section{Enfoques dialógicos en contextos de vulnerabilidad que buscan espacios de democratización de la enseñanza}

La importancia de los elementos sociales, ambientales e interpersonales dentro de los establecimientos educacionales vulnerables, sugiere el desarro- 
Ilo de distintos mecanismos basados en sistemas de identificación y caracterización de las dinámicas de micros-espacios simbólicos al interior del aula (Lundgren, 1992; Torres, 1996). Cuando no se logran detectar las relaciones culturales y simbólicas en las aulas no se consigue garantizar aprendizajes significativos. Los enfoques dialógicos y participativos en establecimientos educacionales bajo contextos de vulneración, permiten establecer aprendizajes que despiertan interés en los estudiantes. La evidencia de los factores socios-económicos no condiciona los aprendizajes. Al momento de democratizar estos aprendizajes y sobre todo las metodologías utilizadas, se genera una mejora en el clima escolar, logrando coordinar con los educantes los tiempos de aprendizaje que garantizan calidad del mismo al interior del aula (Cornejo y Redondo, 2001).

Al abordar la disciplina, en busca de garantizar un ambiente y clima de aprendizaje, es habitual considerar enfoques tradicionales que buscan incentivar sometimiento e imposición de las normas a los educandos. La disciplina genera resistencia cultural adulto vs adolescente/niño y la violencia simbólica rompe todo contexto de respeto y tolerancia en el aula. Los establecimientos educacionales se han convertido en espacios de conservación y reproducción de las estructuras sociales, bajo los parámetros disciplinarios autoritarios. Estos enfoques han dejado de lado los lineamientos participativos, dialógicos y democráticos, que se contraponen a los enfoques más conservadores y tradicionales. Estos mecanismos de reproducción social o desigualdad en los establecimientos y las aulas buscan desarrollar procesos de escolarización preparándolos para el mundo post-industrialización.

Los establecimientos educacionales se re-articulan como lugares de construcción y control de subjetividad. El enfoque participativo, dialógico y democrático posiciona lineamientos de educación que no ayudan a los estudiantes en sus aprendizajes, siendo pertinente a su realidad de contracultura expresada en el aula. La re-construcción de espacios democratizantes permite generar aprendizajes individuales y colectivos que acerquen a los estudiantes a la enseñanza de las matemáticas. La instalación de métodos de enseñanza democratizantes, dialógicos y participativos dentro de las aulas se genera un choque con los lineamientos de carácter relacional de poder que desfavorece la toma de decisiones de los estudiantes. Por este motivo, es fundamental incentivar al interior del aula los métodos dialógicos que rompan las relaciones de poder en el aula. En definitiva, los colegios que abordan a estudiantes en contextos de 
vulneración o de privación socio-económico y cultural experimentan "un primer nivel de exclusión, que podríamos llamar propia de la condición juvenil respecto del mundo adulto. En el caso de la juventud urbano popular nos encontramos con un nuevo nivel de exclusión: la pobreza. En esta medida, la exclusión es doble; se es joven y pobre al mismo tiempo. (Sapiains y Zuleta, 2001, p. 56).

Los enfoques dialógicos que se enmarcan la propuesta de innovación didáctica de este trabajo provocan una aproximación de los análisis epistemológicos de las teorías de la modificabilidad cognitiva estructural y experiencia de aprendizaje mediado. El profesor que se alinea a la mediación del aprendizaje, abre un espectro de flexibilidad didáctica contextualizada en los procesos cognitivos, emocionales y patrones socioculturales. Tal como plantea Tébar (2003) "La decisión del docente de actuar como mediador le trasforma en un interactor creativo, ingenioso e innovador con el educando, independientemente del contenido, del lenguaje o del objetivo inmediato de la experiencia mediada" (p. 14). En definitiva, los lineamientos pedagógicos de carácter dialógico van generando aprendizaje por descubrimiento. Los principios dialógicos generan interrogación como el vehículo de los aprendizajes y fomenta la curiosidad en los estudiantes, adquiriendo las habilida- des y actitudes que son mediadas por el docente (Reibelo, 1998).

La adquisición de habilidades en los estudiantes se resume en los enfoques Vigotskianos, basado en la experiencia de aprendizaje cooperativo. Por lo tanto, siguiendo a Vivas (2010), se comprende que:

\begin{abstract}
a través de su experiencia con el medio pues, como ser social, desde que nace su interacción comunicativa con las personas que la rodean, regula y determina, en última instancia todos sus aprendizajes. Por esto las personas, de cualquier edad, en calidad de grupos humanos, generan estrategias de aprendizaje de tipo interactivo. Es en este tipo de estrategias que se fundamenta el Aprendizaje Cooperativo (p. 34).
\end{abstract}

El proceso desarrollado en el aula busca responder las exigencias de abordar la diversidad en la misma. Por lo tanto, este requiere comprender la importancia de los espacios de sociabilización, valorando los factores internos que existen en el aula. La detección de relaciones inter-subjetivas en la sala instala una sistematización de las acciones didácticas (Medina y Salvador, 2009). La innovación educativa busca abordar las habilidades de razonamiento lógico-matemático, para lo cual requiere "superar los factores 
que lo hacen acomodado o ajustado. Es la lucha por su humanización amenazada constantemente por la opresión que lo ahoga, casi siempre practicada- y eso es lo más- en nombre de su propia liberación" (Freire, 2008, p. 35).

Por lo tanto, las estrategias didácticas para abordar el razonamiento lógico-matemático deberían provocar implicancias contextualizadas en las emociones. Fernández (2010) acota al respecto que:

Los recientes avances en neurociencia ponen de relieve las conexiones entre la emoción, el funcionamiento social, y la toma de decisiones. Estos avances afectan directamente en materia de educación. Los aspectos de la cognición están directamente relacionados y afectados positiva o negativamente por los procesos de emoción. Los aspectos emocionales, el pensamiento y la cognición guardan estrecha relación (p. 10).

Estos elementos, psico-emocionales son herramientas fundamentales para mejorar los aprendizajes y anclar las habilidades lógico-matemáticas. Las principales barreras que se generan para construir aprendizajes anclados y provocar los andamiajes cognitivos, se traducen en las expectativas que tienen los educadores sobre sus estudiantes. Para comprender de mejor manera lo dicho cabe destacar lo propuesto por Román (2006):

Las evidencias siguen siendo contundentes al señalar que la práctica pedagógica de los docentes de escuelas vulnerables está fuertemente asociada a los modelos e imágenes que ellos construyen y reproducen sobre las potencialidades de sus alumnos y alumnas, generándose formas estables e inefectivas en el proceso de enseñanza-aprendizaje. Así entonces, es necesario detenernos un momento en caracterizar la subjetividad de los docentes, respecto de las capacidades y posibilidades que les atribuyen a sus alumnos, en tanto factor relevante en la dinámica del aula, así como en el rendimiento y aprendizajes que alcanzan los niños y las niñas (....). Hay un fuerte consenso entre los docentes al subrayar que los niños de escuelas vulnerables muestran muy pocas habilidades cognitivas y sociales. Por lo general los caracterizan como lentos, carentes de concentración, motivación y poco perseverantes. Del mismo modo, coinciden en describir a estos estudiantes como carentes de afecto y con muy poca ayuda desde el hogar en su proceso escolar (p. 78). 
La construcción de propuesta alternativa enmarcada en los lineamientos dialógicos, participativo y democrático brindan una respuesta a las nuevas interrogantes y problemáticas de la juventud del siglo XXI (Morales, 2012). El aprendizaje centrado en los estudiantes se traduce en un proceso de exploración e indagación de carácter transversal entre estudiante y docente. Este paradigma incorpora y responde a las necesidades de los educadores, incluyendo elementos exteriores de carácter cultural adquiridos previamente por los estudiantes. Este enfoque da respuesta personalizada y prioriza los intereses particulares de los jóvenes en el aula (Ontoria et al., 2006). El proceso pedagógico de carácter horizontal al interior del aula, logra posicionar una perspectiva ecológica de la clase:

Asimismo, ha desencadenado en el estudio de contenido, en alguna manera novedosos, dentro del ambiente escolar, como las variables estrictamente ecológica, los programas educativos y el clima social de la clases. La investigación realizada en torno a estas variables ha servido para roturar un campo de estudio verdaderamente prometedor, como es el examen de las condiciones ambientales específicas en que se realiza el proceso enseñanza-aprendizaje, pues es de esperar que la estructura del escena- rio, el nivel de ruido, o la organización espacial de la clase tengan influencia sobre el comportamiento de los estudiantes (Arancibia, Herrera y Strasser, 2011:37).

Se tomó esta asignatura debido a los bajos resultados que presentaron los estudiantes. Además, existía un conjunto de problemas actitudinales para afrontar las matemáticas. A través de esta propuesta didáctica inclusiva, se buscó acercar los contenidos y las habilidades desde una perspectiva más transversal, dialógica y participativa. El propósito fue mejorar la sana convivencia en la clase y potenciar los aprendizajes de los estudiantes desde se perspectiva y más contextualizada a su realidad.

Para un mayor acercamiento a las matemáticas sin generar rechazo en los estudiantes, el educador debería proponer un sistema de apreciación que le permita analizar, interpretar y apreciar las conversaciones con los educandos y así generar procesos de innovación en el aula (Osorio, 2009). La re-construcción pedagógica dialógica, participativa y democratizante, motiva a los estudiantes a mejorar sus aprendizajes en las matemáticas o razonamiento lógico-matemático. Por ese motivo, es fundamental incentivar en las aulas y establecimientos educacionales la reflexión y repotenciar las prácticas en el aula, por ende, rede- 
finir las sinergias pedagógicas sobre los parámetros praxeológicos.

La praxeología es definida como, "(...) la pedagogía es un saber fruto del encuentro maestro- educando, en términos de la práctica o quehacer profesional; lo que, por una parte, intenta orientar la pedagogía y, por otra, pretende reflexionar y aprender desde y sobre ella" (Juliao, 2014, p. 10). Estos criterios exigen a los profesores de matemática, adquirir un enfoque de

"maestro como un investigador que ejerce su trabajo de modo crítico y autónomo, esto es: como un auténtico pedagogo, lo que requiere una formación praxeológicos que le permita el surgimiento y desarrollo de procesos de reflexión en, desde y sobre la práctica. Se define así la pedagogía como teoría de la acción educativa, en el sentido de una disciplina praxeológicos; es decir, la pedagogía es una teoría a la vez reflexiva y prospectiva, plenamente orientada hacia el utópico futuro, pero fuertemente enraizada en la práctica y la experiencia concreta del presente y, obviamente, enriquecida con los aportes del pasado histórico" (Juliao, 2014, p. 11).

La re-construcción pedagógica concibe la gestión del conocimiento, mediante las experiencias e intervenciones hechas por el educador o equipo multidisciplinario (orientador, psicólogo, educadora diferencial entre otros). En definitiva, este tipo de estrategias busca un trabajo articulado en conjunto en pos de un mejoramiento de las actitudes relacionadas al aprendizaje de los estudiantes. Estas actitudes facilitan la repotenciación de los elementos de liderazgo, monitoreo y gestión del aprendizaje.

Al momento de abordar la enseñanza-aprendizaje de razonamiento lógico-matemático en los estudiantes es posible establecer enfoques horizontales en el aula, desde los prismas éticos y el cuidado de las expresiones simbólicas. El desarrollo de lineamientos didácticos exige la democratización de los espacios de aprendizaje de forma inclusiva, participativa y dialógica (Osorio, 2009). A raíz de los cambios experimentados en la educación, en los contextos globalizantes, se requiere un re-viraje de la didáctica. Este tipo de enfoques pretende entregar a los educantes formas de enseñanza más cercanas a la vida cotidiana, mediante el aporte de los equipos multidisciplinarios, estableciendo una simbiosis disciplinar que se manifieste en el aula (Medina y Salvador, 2009).

Esta propuesta didáctica de carácter inclusivo busca reconstruir los aprendizajes en los estudiantes en contextos vulnerables. Las posturas pedagó- 
gicas verticales y anti-democráticas son experimentadas dentro de este modelo. Por eso, es necesario desarrollar el cognitivismo entendiéndose que "el cognitivismo considera al objeto del conocimiento como perteneciente a un mundo real, el constructivismo es una proposición explicativa de construcción de una rea- lidad personal que se constituye desde las propias experiencias del sujeto epistémico" (Manosalva, 2017, p. 39). Por lo tanto, hay que replantear los modelos de enseñanza-aprendizaje mediante el post-constructivismo como espacio de democratización de las habilidades del razonamiento lógico-matemático.

\section{Desarrollo de la Propuesta}

El diseño de la propuesta de mejoramiento didáctico se posiciona en el modelo didáctico dialógico, participativo e interactivo, en el cual, se propone un enfoque curricular crítico comunicativo (Aubert et al., 2009). Esta herramienta se la teoría de la dialogicidad (Ferrada y Flecha, 2008; Abellán, 2011). En definitiva, esto permite responder a un currículo construido con y desde la comunidad, para superar las desigualdades de los aprendizajes en el aula con carácter de vulnerabilidad (Ferrada, 2001).

La gestión del trabajo colaborativo entre los actores claves del aprendizaje, estudiantes, profesores, educadora diferencial y orientador se enmarcó en un conjunto de reuniones. Estas actividades permitieron diagnosticar las principales dificultades que existía en el aula.

El primer paso estableció un diagnóstico de parte de los estudiantes, en donde se utilizó los espacios de tiempo de la clase de orientación y en el que participaron profesores, jefes y el orientador. En este espacio, se realizó una asamblea con los estudiantes, donde ellos describían y sociabilizaban sus principales problemáticas en el aula. Esta información se registró en una pauta de anotación, que luego fue analizada para buscar la mejor estrategia, bajo una perspectiva de la "comunicación auténtica", proponiendo generar en los profesores total apertura, disponibilidad y evitar el reduccionismo de intereses adulto-céntricos. Así, los estudiantes podrían manifestar libremente como querían recibir la clase, dando paso a la flexibilidad del proceso de aprendizaje (Abellan, 2011). Con esta metodología se buscó llegar a acuerdos con los estudiantes, siendo la base para proyectar cómo podrían ser las clases en un futuro.

El segundo paso hizo un diagnóstico con los docentes y educadoras 
diferenciales o quienes tuvieron participación en el aula. Luego de estas actividades, se levantó los relatos más significativos y se identificó las principales problemáticas en el aula. Al finalizar la gestión del trabajo colaborativo, se propuso acuerdos y responsabilidades. Esta información, generó un registro de las distintas opiniones de los actores.

El tercer paso diseñó una propuesta didáctica donde se recopiló todas las opiniones de los actores educativos y se temporalizó para su ejecución. Además, se presentó la propuesta a los estudiantes en la clase de orientación, donde ellos podían hacer observaciones y decidir si les interesaba con esta última opinión la planificación propuesta.

Este diseño buscó generar un espacio de diálogo participativo que fuera en contra de los modelos tradicionales de la educación (Prieto y Duque, 2009). Cabe mencionar lo acotado por Velasco y González (2008):

“En cuanto atañe al problema del manejo del diálogo en la práctica educativa, las críticas a la educación tradicional se han centrado fundamentalmente en la escasa o nula presencia del diálogo en el aula, entendido éste como intercambio comunicativo equitativo y respe- tuoso en una interacción equilibrada" (p. 462).

El modelo didáctico dialógico/-participativo es adecuado en los aprendizajes de los contextos de alta vulneración social y cultural. Además, permite generar y nivelar, expectativas y aprendizajes en los estudiantes/docente (Andreucci, 2012; Flecha et al., 2004). El trabajo colaborativo de los distintos actores de la educación incentiva los niveles de pertenencia y mejora los ambientes de aprendizaje, siendo considerado el estudiante como uno más del aprendizaje y no un simple receptor (Ferrada, 2008). Por lo tanto, el diseño dialógico/participativo sugiere instalar un razonamiento de mejora participativa por sobre las razones de poder adulto-céntrica que en muchos casos genera rechazo y des-aprendizaje. (Velazco y González, 2012; Ferrada, 2008).

A raíz de estas herramientas, se construyó la propuesta que designó tareas a los distintos grupos de trabajo (comisiones heterogéneas). Fomentar el diálogo y la participación se posiciona en la recuperación de la base de la pedagogía Zona Desarrollo Próximo (ZDP) de Vygotsky (1979). Esta herramienta no se limita a la guía del docente, sino se convierte en un mediador del aprendizaje (Tébar, 2009). 


\section{DETECCIÓN DE LA PROBLEMÁTICA}

Las principales problemáticas detectas en los cursos intervenidos fueron la poca motivación por aprender las matemáticas, el ambiente tóxico del aula, conflictos entre los estudiantes y el/los docentes, y poca participación de los estudiantes en clase.

En las distintas reuniones con los docentes de matemática y específicamente de los cursos intervenidos, se mencionó que los estudiantes de sexto básico tenían una baja motivación por la asignatura. Esto se traducía que los estudiantes no tomaban notas y no realizaban los ejercicios del docente. A raíz de esto, los estudiantes no tenían buenas calificaciones en las distintas actividades y pruebas que realizaban. Cuando se conversaba con los estudiantes sobre las principales problemáticas, las principales ideas que mencionaron se relacionaban con el hecho de que estaban desmotivados porque no entendían la materia. Por ese motivo, no querían ingresar a clase y preferían conversar antes que estudiar, hacer desorden en la sala de clase y escuchar música.

En las conversaciones que se realizaron en la clase de orientación sobre la problemática que estaban experimentando conflictos en el aula. Los principales elementos del ambiente tóxico del aula, estaban relacionado con la poca participación en clase tenían con el docente y la actitud que ellos tenían con la asignatura. Estos factores sumados a los conflictos que se fueron generando entre el docente y los estudiantes que término provocando un clima de aula tóxico que no permitió generar aprendizaje.

El conflicto que se generaba con el/los profesores, especialmente con el docente de matemática, provoco que los procesos del aula no se llevaran a cabo como estaba planificado esto generó una tensión el aula. Esta tensión generada en el aula entre profesores y estudiantes no permitió que se produjera un aprendizaje significativo. Por otro lado, los profesores, también comenzaron a sentir malestar en realizar clase en los cursos. En varias ocasiones, se tuvieron que realizar mediaciones para mejorar las relaciones interpersonales por el equipo externo (orientador, inspector general y/o educadoras diferenciales) para optimizar las relaciones entre profesor y estudiantes. El docente realizaba todos los esfuerzos para tener una sana convivencia, sin embargo, existía un rechazo por la poca flexibilidad de los procesos de aprendizaje.

A raíz del conjunto de problemática que experimentaban los cursos, se generó una actitud más autoritaria y vertical en el contexto del aula. Los pro- 
cesos en el aula, comenzaron a provocar sentido de poca participación en el aula. Los estudiantes manifestaron que querían mayor democratización en la enseñanza. Sin embargo, luego de varios en- cuentros dialógicos con los estudiantes, se asumieron niveles de participación. Estas decisiones se contraponían a las posturas verticales y poco democráticas de la enseñanza.

\section{METODOLOGÍA DE LA IMPLEMENTACIÓN DEL DISEÑO DIDÁCTICO}

La metodología del diseño y ejecución de la propuesta se enmarcó en la Investigación Acción Participativa (Sandoval, 2002; Becerra, Moya, 2010). La misma se dividió de la siguiente manera:

Primer momento: se estableció reunión con los estudiantes de $6^{\circ}$ básicos, con la finalidad de potenciar las estrategias, generar niveles de participación, compromiso y colaboración en la propuesta.

Segundo momento: se gestionó la coordinación con los docentes, con la finalidad de abrir un espacio de diálogo pedagógico y establecer consensos para ejecutar la propuesta.

Tercer momento: se establecieron reuniones de gestión con los profesores jefes, con la finalidad de establecer lineamientos y comunicación de los actores educativos, debido a que ellos son los que tienen mayor conocimiento de sus cursos.

Cuarto momento: se establecieron reuniones de gestión con el equipo directivo, con la finalidad de establecer lineamientos comunicativos y apoyo en la generación de los distintos espacios del establecimiento para generar las propuestas.

\section{Descripción del Diseño didáctico:}

Luego de las distintas reuniones de gestión, se calendarizó la ejecución del diseño de innovación en los distintos cursos:

El primer paso fue en la sala de clase con los educadores y los estudiantes en donde se presentó la propuesta, entregándose las instrucciones y los requisitos mínimos de conducta para afrontar este desafío. En esta propuesta se buscó que cada estudiante y grupo de trabajo fuera responsable de cada estación de aprendizaje. Cada grupo que participaría, debería luego finalizado el ejercicio, ordenar para que los siguientes grupos puedan participar.

En el segundo paso, todo el curso fue al patio techado que tiene el Cole- 
gio. En este espacio, se les presentaron las distintas estaciones de aprendizaje/ ejercitación o circuitos donde cada mesa tendría distintos ejercicios matemáticos.

El tercer paso, en las mesas los estudiantes tenían 2 minutos para resolver el ejercicio de selección múltiple, que fue elaborado por el profesor de matemática. Además, dentro del circuito se organizaron tres grupos, que debían recorrer por cada una de las estaciones utilizando la técnica de postas.

El cuarto paso, cuando los estudiantes van resolviendo los ejercicios, debieron correr a la otra mesa para que el compañero salga a resolver el ejercicio en las otras mesas.
En el quinto paso, los estudiantes van recorriendo las distintas mesas, tuvieron que responder preguntas de selección múltiples y finalizan el recorrido en la mesa número 5 donde tenían que botar unos tarros.

En el sexto paso, los educadores recogen los ejercicios que luego son revisados por el profesor.

Y el séptimo paso, se hace ingresar al curso a la sala, en donde se presenta los resultados de las respuestas correctas y se solicita una evaluación de la actividad por parte de los estudiantes.

A continuación, en la Figura 1 se esquematiza la propuesta de innovación educativa.

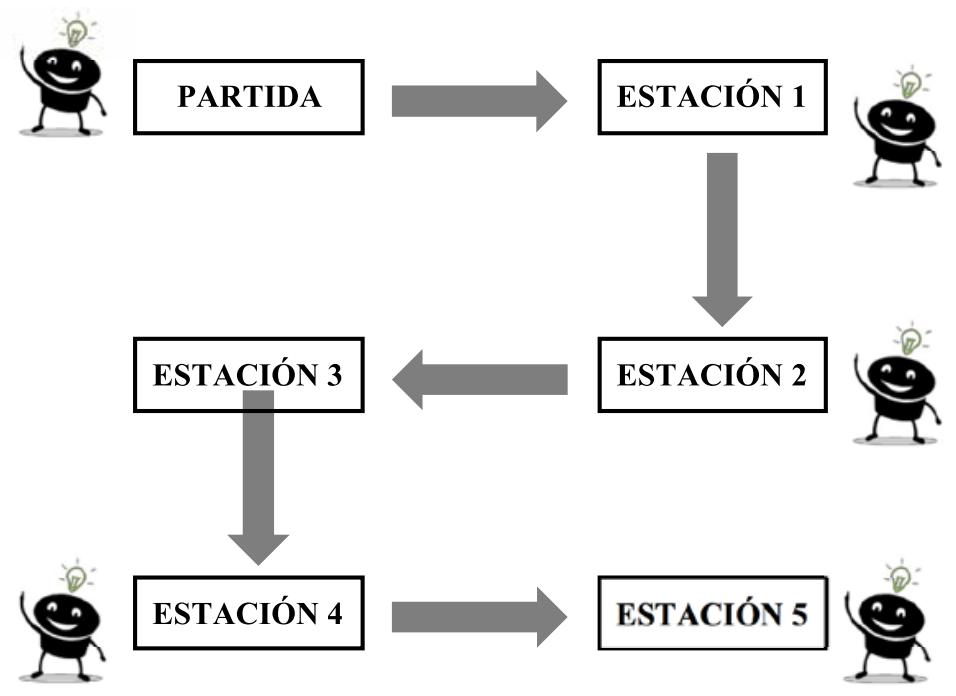

Figura 1. Descripción de los pasos de la propuesta de innovación educativa 


\section{RESULTADOS Y DISCUSIÓN}

La democratización de los espacios de aprendizaje se traduce en un mayor vínculo con los contenidos, habilidades y actitudes de aprendizaje que potencia la adquisición de los pensamientos de razonamiento lógico y matemático. Cuando se empodera a los estudiantes en cómo quieren aprender, la respuesta a una mejor disposición para adquirir nuevos conocimientos provoca que ellos se auto-regulen y, así, se obtiene el objetivo esperados del desarrollo de habilidades. Ejemplo:

Al principio estuvimos un poco desordenados, pero fue súper bacán hacer una clase de matemática de esta forma, siento que ahora entiendo más los ejercicios.

María Santos, $6^{\circ} \mathrm{A}$

Los estudiantes están ávidos de ser actores activos de su propio aprendizaje. Sin embargo, la mayoría de las veces se terminan posicionando los enfoques tradicionales de carácter vertical. Estos no permite profundizar el desarrollo de otros tipos de aprendizaje de carácter de habilidades blandas, debido que se privilegios los aspectos escolarizantes y no los educativos. Los estudiantes expresan la necesidad de que las clases de matemáticas sean más interactivas e innova- doras. Un ejemplo de su percepción se expresa de la siguiente manera:

Fue súper buena la actividad. Nos gustó mucho. Podríamos hacer más clases de este tipo.

Estudiante Nissette González, 6 B

Por otro lado, la percepción de los estudiantes está relacionada con romper los esquemas tradicionales que muchas veces se omite. Debido a la existencia de garantizar una perspectiva de control y regulación de los espacio pedagógicos compartidos. Se los convierte en mecanismos de sometimiento bajo los prisma de forma buena mano obra. Por este motivo, no existen los espacios democratizados si liberalizamos los procesos de enseñanza-aprendizaje que el estudiante propone realizar en sus proceso de aprendizaje en el aula. Ejemplo de aquello:

¿Porque no hacemos más clases en el patio o gimnasio? Son súper entretenidas. El único problema, profesor, es que no todos nos portamos bien; pero, al final, todos participamos de la actividad.

Belén Riquelme, $6^{\circ} \mathrm{B}$ 
Cuando se generan los espacios de participación a nivel transversal, los estudiantes modifican las actitudes conductuales. Esto se traduce en un mejoramiento del ambiente del aula y/o ambientes de aprendizaje, debido que los contextos socio-culturales que experimentan los estudiantes de carácter de privación y de un fuerte autoritarismo simbólico. Por este motivo, es fundamental instalar en los colegios un espacio de participación y democratización de los procesos de aprendizaje, traduciendo en un mejoramiento de los climas de aula y/o ambientes de aprendizaje. Ejemplo de la percepción que tuvieron los estudiantes:

Fue súper bacán la actividad. Nos parece que deberíamos seguir haciendo esta actividad, además nos portamos mucho mejor que en la sala.

Lucas Cifuentes, $6^{\circ} \mathrm{C}$

Desde el punto de vista pedagógico, el modelo didáctico dialógico, participativo y democrático, permitió dar cuenta de la vinculación entre el aprendizaje instrumental y el social. Al interior de cada actividad programada, se favoreció la diversificación de estrategias de aprendizaje, considerando la heterogeneidad de los estudiantes y aasegurando la atención personalizada. Mediante la formación de grupos pequeños con un colaborador de aprendizaje, fue posible dar cuenta de un grupo diversificado de agentes sociales que trabajan voluntariamente como colaboradores de aprendizaje y que matizan las interacciones del estudiantado, a la vez que constituyen otros referentes pedagógicos distintos al profesor. El profesor, basado en esta herramienta pedagógica, dio cuenta de su rol profesional en la coordinación del trabajo pedagógico entre todos los colaboradores y todos sus estudiantes, buscando un rol protagónico en el estudiante. Además, se fomentaron valores constructivos y colaborativos en su aprendizaje y en el de sus pares, en forma permanente. 


\section{ANÁLISIS}

Los estudiantes hacen el recorrido de una mesa a otra. Primero, corrieron y llegaron a la mesa $\mathrm{N}^{\circ} 1$; el recorrido de la mesa $\mathrm{N}^{\circ} 1$ a la $\mathrm{N}^{\circ} 2$. El estudiante, debió ir a la siguiente mesa ( $\left.\mathrm{N}^{\circ} 3\right)$ en una carrera del saco, en donde debían llevar una pelota con una cuchara. En el recorrido de la mesa $N^{\circ} 3$ a la $N^{\circ} 4$, los estudiantes debieron apuntar a las botellas con un aro; el recorrido de la mesa $\mathrm{N}^{\circ} 4$ a la $\mathrm{N}^{\circ} 5$ se realizó corriendo, sacando un dulce de un plato de agua con la boca. Finalmente, en el recorrido de la mesa $\mathrm{N}^{\circ} 5 \mathrm{a}$ la meta, se debió botar los tarros con una pelota de calcetines.
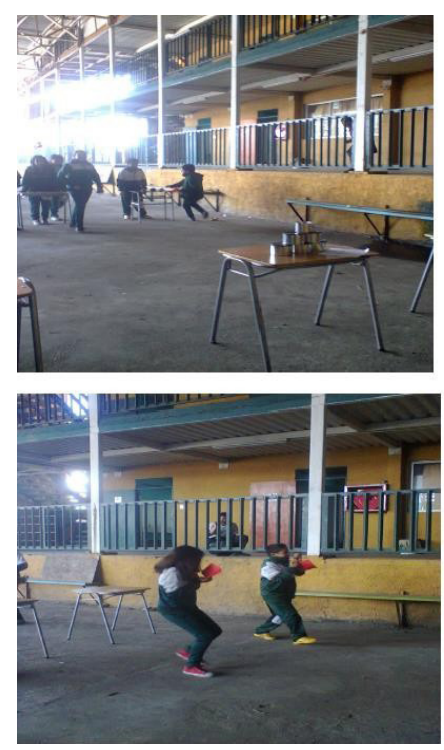

Esta propuesta dialógica, participativa y democratizadora de los proceso de enseñanza- aprendizaje se utiliza la perspectiva bio-psico-social. Esta perspectiva posiciona los factores biológico, particular y social, mediante los prismas holísticos. Esto último utiliza las variables contextuales, ambientales y culturales del entorno social de cada estudiante que permiten mayor participación de los educandos en sus procesos de enseñanza-aprendizaje.

A continuación, se adjunta en la Figura 2 material fotográfico de evidencia del diseño de innovación.
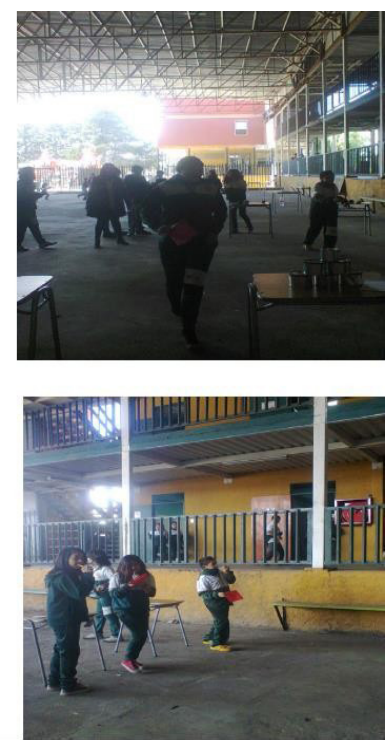


INNOVACIÓN DIDÁCTICA INCLUSIVA BASADA EN LA PEDAGOGÍA DIALÓGICA PARTICIPATIVA E INTERACTIVA APLICADA AL ÁREA DE MATEMÁTICA EN ESTUDIANTE \|\|$_{\| \downarrow}$ VULNERABLE BASADA EN UN ESTUDIO DE CASO
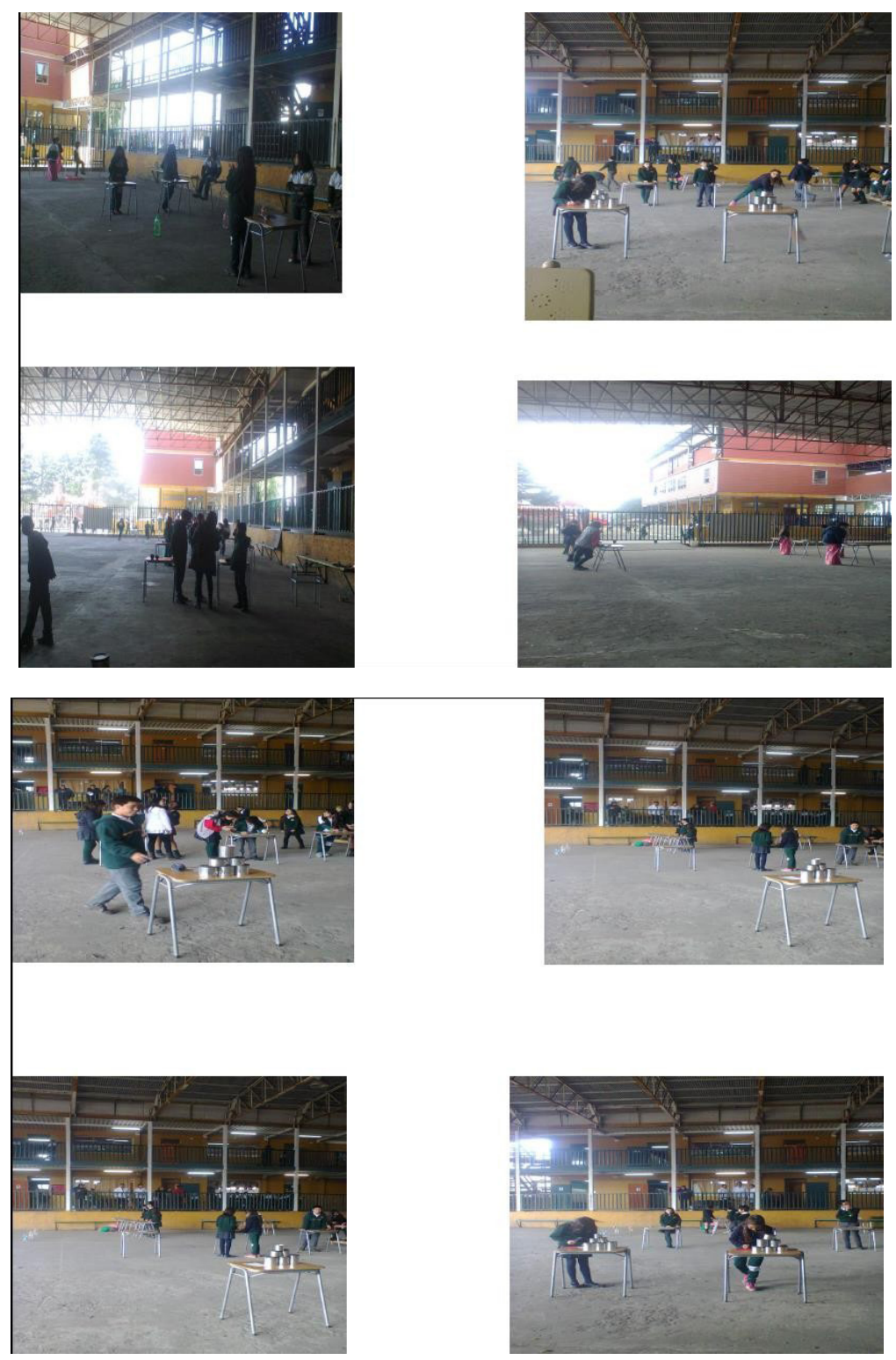

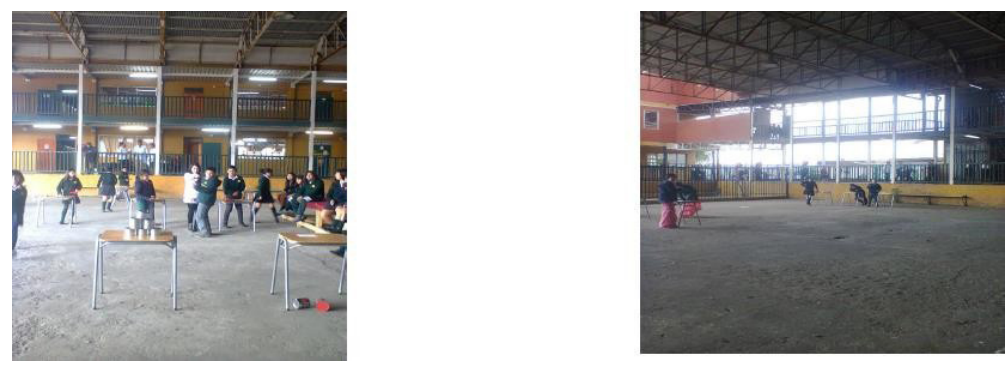

Figura 2. Material fotográfico que evidencia la propuesta de innovación educativa

\section{CONCLUSIONES}

Las principales conclusiones de este trabajo mencionan la importancia de fomentar espacios de diálogo de carácter transversal. Esta propuesta didáctica de innovación se sustenta en la pedagogía dialógica participativa e interactiva y es una experiencia exitosamente aplicada. A partir de estas herramientas, se puede obtener resultados de mejora en el ambiente del aula, escuchando el interés de los estudiantes, fomentando un trabajo colaborativo multidisciplinario $y$, sobre todo, mejorando los aprendizajes en las distintas asignaturas, como fue el caso exitoso de la Yincana de las Matemáticas. El valor de este trabajo es avala- do por las opiniones de los estudiantes, de quienes se describieron la valoración de cómo ellos quieren aprender una asignatura que en su momento tenía niveles de complejidad.

Este enfoque dialógico participativo, inclusivo y didáctico, facilitó la instalación de un trabajo colaborativo para potenciar los aprendizajes de los estudiantes. Además, genera niveles de implicancia de los actores educativo, a raíz de que nace desde los propios interés de los actores educativos, provocando una mayor profundización del cómo y el para qué de la asignatura de las matemáticas. 


\section{BIBLIOGRAFÍA}

Abellán, A. (2011). Teoría dialógica de la comunicación: devolver al hombre con el hombre al centro de la investigación. Revista Comunicación y Hombre. 7: 214-225.

Andreucci, P. (2012). el talento: una construcción en y desde la pedagogía dialógica. Psicoperspectivas. Individuo y Sociedad, 11 (2): 185-205. Arancibia, V, Herrera, P y Strasser, K (2011). Manual de Psicología Educacional. Ediciones Universidad Católica de Chile, Santiago, Chile.

Aubert, A, Garcia, C., Racionero, S. (2009). El aprendizaje Dialógico. Cultura y Educación, 2009, 21 (2), 129-139.

Becerra, R, Moya, A. (2010) Investigación- acción participativa, crítica y transformadora. Un proceso permanente de construcción. Integra Educativa III(2): 132-156.

Cornejo, R. y Redondo, J. (2001). El clima escolar percibido por los alumnos de enseñanza media: Una investigación en algunos liceos de la Región Metropolitana. Última década, 9(15), 11-52.

Fernández Bravo, J. A. (2010): Neurociencias y enseñanza de la matemática. Revista Iberoamericana de Educación/Revista Ibero-Americana de Educção (RIE), Versión Digital 51(3),
Ferrada, D. (2001). "Comunidades de entendimiento": una propuesta educativa desde la perspectiva crítica comunicativa del currículum. Pensamiento Educativo. 29, 297-317.

Ferrada, D. (2008). El principio de emocionalidad- Corporeidad: Un complemento al modelo de aprendizaje ideológico. Educere, 13 (44), $1316-4910$.

Ferrada, D., Flecha, R. (2008) el modelo dialógico de la pedagogía: un aporte desde las experiencias de comunidades de aprendizaje. Estudios pedagógicos XXXIV(1), 41-61.

Flecha, R., Vargas, J., Davila, A. (2004). Metodología comunicativa crítica en la investigación en ciencias sociales: La investigación Workaló. Lan harremanak,11(II), 21-33.

Freire, P. (2008). La educación como práctica de la libertad. Editorial XXI, segundo edición, Buenos Aires, Argentina..

Juliao, G. (2014). Pedagogía praxeológicos y social: Hacia otra educación. Editorial Centro de Pensamiento Humano y Social (cphs). Bogotá, Colombia,

Manosalva, S. (2017). El sujeto del aprendizaje: un esbozo epistemológi- 
co desde la filosofía y psicología educativa. Ediciones CEU, Santiago de Chile.

Medina, A. y Salvador, F. (2009) Didáctica General. Editorial Pearson Educación, Madrid, España,

Medrano, C. (1995). La interacción entre compañeros: el Conflicto sociocognitivo, el aprendizaje cooperativo y la tutoría entre iguales. Rvta. Interuniversitaria de Formación del Profesorado, 23, 177-186. Molina, W. y Sandoval, M. (2006). Cultura escolar y cultura juvenil: La (re) construcción simbólica del espacio escolar en la mutación cultural., Revista Temas Sociológicos No $11 \cdot 2006 \cdot$ pp. $103-123$

Morales, P. (2012). Hacia intervenciones sociales democratizantes: aportes desde el prisma discursivo de jürgen habermas. Revista Trabajo Social, 83, 7-22.

Ontoria, A., Gómez,J., Molina, A. y Luque, A. (2006) Aprendizaje centrado en el alumno. Metodología para una escuela abierta. Narcea, S.A. de Ediciones, Madrid, España,.

Osorio, J. (2009). Lo próximo-complejo: enfoque eco-reflexivo en educación y política ciudadana. Editorial Universidad Bolivariana, Santiago, Chile

Prieto, O., Duque, E. (2009) El aprendizaje Dialógico y sus aportaciones a la teoría de la educación. Teoría de la educación. Educación y Cultura en la sociedad de la información, 10(3), 7-30.

Reibelo, J. (1998). Método De Enseñanza. Aprendizaje Para La Enseñanza Por Descubrimiento. Aula abierta, 71, 123-147.

Román, M., Díaz, E. (2009). Inteligencia escolar. Aplicación al aula. Editorial Conocimiento, Santiago, Chile,

Román, M. (2006). El Desafío De Aprender A Leer, Comprender Y Razonar En Escuelas Vulnerables. Rev. Pensamiento Educativo, 39(2), 69-86

Román, M. y Díaz, E. (1999). El Currículum como desarrollo de procesos cognitivos y afectivos. Revista Enfoque Educacionales, 2(2), 48-67.

Salazar, G. (2011). En el nombre del poder popular constituyente (Chile, siglo XXI).Editorial LOM, Santiago, Chile,

Sandoval, C. (2002). Investigación Cualitativa. ARFO, editorial e impresores Ltda. Bogotá, Colombia,

Sapiains, R. y Zuleta, P. (2001). Representaciones sociales de la escuela en jóvenes urbano populares desescolarizados. Última década, 9(15), 53-72

Tébar, L (2003) El profesor mediador del aprendizaje. Editorial Conoci- 
miento, Santiago, Chile,

Van Dijk, T. (1994). Conferencias de Teun Van Dijk. Cuaderno, 2(2),. Velasco, J; González, L (2008). Sobre la teoría de la educación dialógica. Educere, 12(42), 461-470.

Vivas, A. (2010). Estrategia de Aprendizaje. Gondola, 5(1), 27-37.
Torres, J. (1996). El curriculum oculto. Editorial Morata. Madrid, España, Rodríguez, M. (1997). Hacia una didáctica crítica. Editorial La muralla. Madrid, España,

Lundgren, U.P. (1992). Teoría del curriculum y escolarización. Editorial Morata. Madrid, España, 